\title{
Iterative Multipacket Detection for High Throughput Transmissions in OFDM Systems
}

\author{
Nuno Souto, Rui Dinis, Member, IEEE, João Carlos Silva, and Paulo Carvalho
}

\begin{abstract}
This paper presents a multipacket detection technique for dealing with packet collisions in OFDM schemes (Orthogonal Frequency Division Multiplexing). Instead of discarding collided packets as it is done in traditional MAC approaches (Medium Access Control) additional packets are transmitted and separated using Multiple Input Multiple Output (MIMO) based techniques. Reliable detection and high throughputs can be achieved as long as different interleavers are used for different retransmissions. We also include a method for estimating the users involved in the collision.
\end{abstract}

Index Terms-Code acquisition and synchronization, codedivision multiple access (CDMA), optical CDMA, optical orthogonal codes (OOCs).

\section{INTRODUCTION}

$\mathbf{P}$ ACKET collision occurs when different users try to access simultaneously a given physical channel. The conventional approach is to discard all blocks involved in the collision and retransmit them again. To reduce the chances of multiple collisions each user transmits in the next available slot with a given probability. Therefore, if two packets collide we need at least three time slots to complete the transmission, which results in throughput loss.

To improve throughput, a TA (Tree Algorithm) combined with a SIC (Successive Interference Cancellation) scheme was proposed in [1]. Within the SIC-TA scheme, the signal associated with a collision is not discarded. Instead, once we receive with success the packet of one of those users, we can subtract it from the signal with collision and recover the packet from the other user. A collision involving two packets requires only one additional time slot to complete the transmission, unless there are multiple collisions. However, in SIC-TA decision errors may lead to a deadlock [2] and we do not take full advantage of the information in the collision. In [3] a multipacket detection technique was proposed where all users involved in a collision of $N_{P}$ packets retransmit their packets $N_{P}-1$ times, each one with a different phase rotation to allow packet separation. This technique is only suitable for

Paper approved by S. K. Wilson, the Editor for Multicarrier Modulation of the IEEE Communications Society. Manuscript received May 19, 2008; revised October 9, 2008, February 17, 2009, and March 12, 2009.

N. M. B. Souto and J. C. M. Silva are with the ISCTE/Instituto de Telecomunicações, Av Forças Armadas, Ed. ISCTE, 1649-026, Lisbon, Portugal (e-mail: \{nuno.souto, joao.carlos.silva\}@1x.it.pt).

R. Dinis is with DEE FCT-UNL (Department of Electrical Engineering of Faculdade de Ciências e Tecnologia da Universidade Nova de Lisboa) and the Instituto de Telecomunicações, Av. Rovisco Pais 1, Torre Norte, 1049 Lisbon, Portugal (e-mail: rdinis@ist.utl.pt).

P. Montezuma is with DEE FCT-UNL (Department of Electrical Engineering of Faculdade de Ciências e Tecnologia da Universidade Nova de Lisboa) and UNINOVA (Instituto de Desenvolvimento de Novas Tecnologias), Monte da Caparica, Portugal (e-mail: pmc@uninova.pt).

Digital Object Identifier 10.1109/TCOMM.2010.02.080247 flat-fading channels (there are phase rotations that might lead to an ill-conditioned packet separation) and it is difficult to cope with channel variations during the time interval required to transmit the $N_{P}$ variants of each packets (the same was also true for the SIC-TA technique of [1]). A modified version of [3] appropriate for time-dispersive channels was proposed in [4], although the receiver complexity can become very high for severely time-dispersive channels. In [5] several multipacket detection techniques are described in addition to showing the advantages of a jointly designed PHY and MAC layer.

A promising method for resolving multiple collisions was proposed in [6] for SC modulations (Single Carrier) with FDE (Frequency-Domain Equalization). Since this technique is able to cope with multiple collisions, the achievable throughputs can be very high. However, the receiver complexity can be rather high since a pair DFT/IDFT is required for each packet and each iteration. In this paper we extend the approach in [6] to OFDM [7] based systems. To detect all the simultaneously transmitted packets we propose an iterative multipacket receiver capable of extracting the packets involved in successive collisions. To be effective the receiver requires uncorrelated channels for different retransmissions which could compromise its use in quasi-stationary channels. However, due to the use of OFDM, simply sending different interleaved versions of the data blocks in different retransmissions can ensure the reliability of the technique. An important problem with multipacket detection schemes is the estimation of the users involved in a collision (as well as their channel frequency responses). We propose a suitable method to obtain this.

\section{System Description}

Let us consider an OFDM scheme with $N$ subcarriers where each user can transmit a packet in a given time slot. If $N_{P}$ users decide to transmit a packet in the same time slot a collision involving $N_{P}$ packets will result. The receiver (typically the BS - Base Station) then requests all users to retransmit interleaved versions of their packets $N_{P}-1$ times (and informs in which time-slots, to avoid collisions with new users). The basic idea is to use all the received transmission attempts to separate the colliding packets. In fact, our system can be regarded as a MIMO system where each input corresponds to a given packet and each output corresponds to a different version of the collision. Therefore, to accomplish reliable detection at the receiver it is important that the correlation ${ }^{1}$ between

\footnotetext{
${ }^{1}$ Clearly, using different symbol-level interleavers before mapping the coded symbols in the OFDM subcarriers is formally equivalent to interleave the channel frequency response for different subcarriers. For a given subcarrier, this reduces the correlation between the channel frequency response for different retransmissions.
} 
multiple received retransmissions (i.e., multiple versions of each packet involved in the collision) is as low as possible. To overcome the problem of high correlation present in slowvarying channels, we can take advantage of the nature of OFDM transmissions over severely time-dispersive channels where the channel frequency response can change significantly after just a few subcarriers. As a result the channel frequency response for subcarriers that are not close can be almost uncorrelated. By simply applying a different interleaving (which we will call symbol interleavers) to the modulated symbols in each retransmission it is possible to reduce the correlation between multiple received retransmissions. The receiver detects the packets involved in the collision as soon as it receives $N_{P}$ different versions of the colliding packets. For simplicity we assume that different packets arrive simultaneously, which means that some coarse time-advance mechanism is required, although some residual time synchronization error can be absorbed by the cyclic prefix.

The structure of the proposed iterative receiver is shown in Fig. 1. As with conventional OFDM receivers, the signals associated with different retransmissions are sampled, the cyclic prefix removed, converted to the frequency domain through an appropriate size- $N$ DFT operation and de-interleaved according to the retransmission to which they belong. The sequences of samples associated with all retransmissions are used for detecting all the packets inside the Multipacket Detector which can apply several different well known methods employed in MIMO decoding [8]. In this study we will consider the MMSE criterion (Minimum Mean Squared Error) for the first iteration and an interference canceller (IC) [8] for the next three iterations. The Decision Device uses the output from the channel decoder to produce soft-decision estimates of the code symbols. The Transmitted Signal Rebuilder reconstructs the transmitted symbol sequences which are then used for a refinement of the channel estimates and also for improvement of the multipacket detection task for the subsequent iteration.

To achieve coherent detection at the receiver, known pilot symbols are periodically inserted into the data stream using a spacing of $\Delta N_{T}$ OFDM blocks in the time domain and $\Delta N_{F}$ subcarriers in the frequency domain. To avoid interference between pilots of different users, FDM (Frequency Division Multiplexing) is employed, which means that pilot symbols cannot be transmitted over the same subcarrier by different users (the minimum allowed spacing in the frequency domain is $\left(\Delta N_{F}\right)_{\min }=N_{p, \max }$, where $N_{p, \max }$ is the maximum number of users that can try to transmit simultaneously). The frequency channel response estimates for each user can be obtained iteratively using the sequence of steps described in [8].

\section{Detection of Users Involved in a Collision}

One of the difficulties of employing multipacket detector schemes, lies in the determination of which users have packets involved in the collision, which is crucial for the reliability of the packet separation. Detecting a lower number of users, in addition to missing the undetected users (which also happens in conventional schemes), will result in an insufficient number of retransmissions to reliably extract the others. On the other hand, in the case of false detections more retransmissions will be requested than those really required, wasting resources (this problem also arises in conventional schemes) and degrading the joint packet separation due to the attempt to extract packets that do not exist. In the following we propose a simple detection method that can be combined with the multipacket detection approach described previously. The method considers the use of OFDM blocks with pilots multiplexed with data, as described in the previous section. Since each user $p$ has a specific subset of subcarriers reserved for its pilot symbols the receiver can use them to estimate whether the user is transmitting a packet or not. First it computes

$$
Y_{p}=\sum_{k, l}^{N_{\text {pilots }}}\left|R_{k, l}^{1}\right|^{2}, p=1, \ldots, N_{p, \max }
$$

for all users, where $N_{\text {pilots }}$ is the total number of pilots used inside the sum and $R_{k, l}^{1}$ is a received sample from the first transmission attempt on sub-carrier $k$ and OFDM block $l$ containing a pilot symbol of user $p$. $Y_{p}$ can be compared with a threshold $y^{t h}$ to decide if a user is active or not. Assuming a worst-case scenario where any incorrect detection of the number of users results in the loss of all packets, the gross simplified system throughput (not taking into account bit errors in decoded packets) is given in [[3], eq. (21)]. Given that the objective is to improve the system throughput then the threshold value, yth , that maximizes [[3], eq. (21)] can be easily shown to occur approximately (assuming low false alarm and missed detection probabilities) when

$$
p_{1}(y)=p_{2}(y) \frac{N_{p, \max }-P_{e}\left(N_{p, \max }-1\right)}{\left(N_{p, \max }-1\right) P_{e}},
$$

where $p_{1}(y)$ is the probability density function (PDF) of (1) when $R_{k, l}^{1}=N_{k, l}^{1}$ while $p_{2}(y)$ is the PDF when $R_{k, l}^{1}=$ $S_{k, l}^{p, p i l o t} H_{k, l}^{p, 1}+N_{k, l}^{1}$, with $H_{k, l}^{p, 1}$ denoting the complex valued channel frequency response in the first transmission attempt by user $p, N_{k, l}^{p}$ the corresponding channel noise and $S_{k, l}^{p, p i l o t}$ a pilot symbol. $P_{e}$ is the probability of a user's buffer being empty at the beginning of a transmission slot. In the presence of additive white Gaussian noise (AWGN) $p_{1}(y)$ corresponds to an Erlang distribution [12], i.e.,

$$
p_{1}(y)=\frac{y^{N_{\text {pilots }}-1} \exp \left(-\frac{y}{\mu_{1}}\right)}{\mu_{1}^{N_{\text {pilots }}}\left(N_{\text {pilots }}-1\right) !} u(y),
$$

where $\mu_{1}=E\left[\left|N_{k, l}^{1}\right|^{2}\right]$ and $u(x)$ represents the unit step function (i.e., $u(x)=0$ for $x<0$ and 1 for $x>0$ ). Regarding the second PDF, since the receiver does not have a priori knowledge about the PDP (Power Delay Profile) of each user, it does not know the correlation between the different $H_{k, l}^{p, 1}$ present inside the summation. For that reason, we opted to employ a threshold located in the middle of those obtained assuming two extreme cases: uncorrelated channel frequency response coefficients and constant channel frequency response coefficients.

\section{A. Uncorrelated Channel Frequency Response}

If $H_{k, l}^{p, 1}$ can be assumed uncorrelated for different $k$ and $l$ (for example a severe time-dispersive channel) then the 


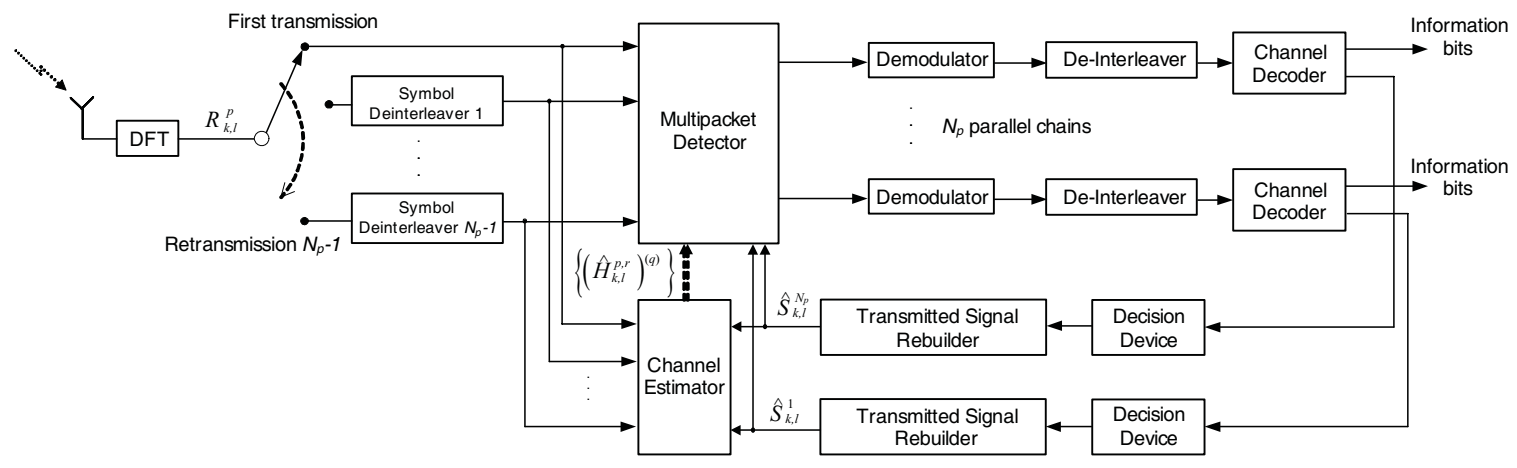

Fig. 1. Iterative receiver structure.

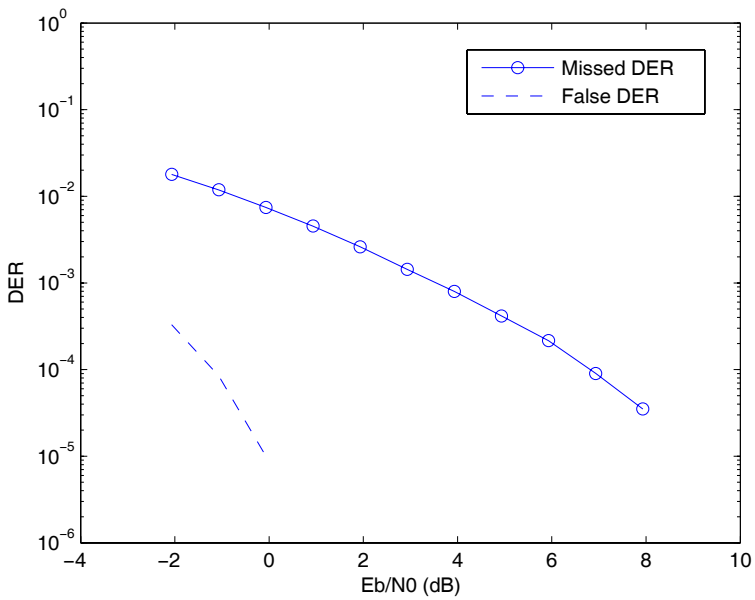

Fig. 2. Detection error rate for $N_{p, \max }=4$.

decision variable $Y_{p}$ also follows an Erlang distribution with component mean $\mu_{2}=\left|S_{k, l}^{p, p i l o t}\right| E\left[\left|H_{k, l}^{p, 1}\right|^{2}\right]+N_{0}$. From (2) it is simple to show that the threshold is given by

$$
y^{\text {th }}=\frac{N_{\text {pilots }} \ln \left(\frac{\mu_{2}}{\mu_{1}}\right)-\ln \left(\frac{N_{p, \max }-P_{e}\left(N_{p, \max }-1\right)}{\left(N_{p, \max }-1\right) P_{e}}\right)}{\frac{1}{\mu_{1}}-\frac{1}{\mu_{2}}} .
$$

\section{B. Constant Channel Frequency Response}

When the channel is not dispersive in time, $H_{k, l}^{p, 1}$ will be almost constant for different $k$ and $l$ and the decision variable $Y_{p}$ will correspond to a sum of correlated exponential variables. From [9], the respective PDF expression can be written as (5) (or alternatively as a single gamma-series [[10], eq. (5)]), where $\rho$ is the correlation coefficient between different received samples (assumed constant) and ${ }_{1} F_{1}(\cdot, .,$. is the confluent hypergeometric function [11]. In this situation the weighted intersection of PDFs (3) and (5) (threshold $y^{t h}$ ) can be easily found numerically.

\section{NUMERICAL RESUlTS}

We start by evaluating the active users detection approach described in Section III. The channel impulse response was characterized by the Vehicular A [13] based PDP (Power Delay Profile) (similar results would be obtained for other severely time-dispersive channels) with Rayleigh fading in the

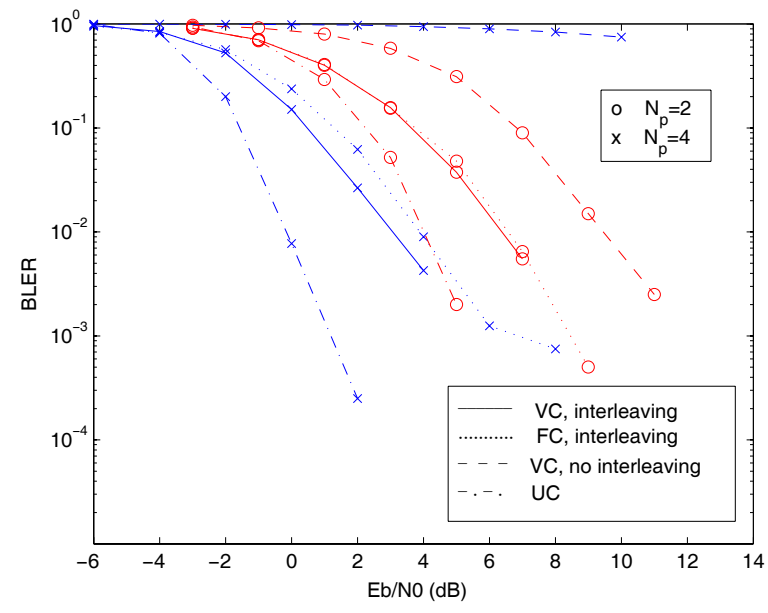

Fig. 3. Impact of using different interleavings for different retransmissions.

different paths. We used $\mathrm{N}=256$, a subcarrier spacing of 15 $\mathrm{kHz}$, QPSK modulation, a pilots spacing of $\Delta N_{F}=N_{p \text {, max }}$ and $\Delta N_{T}=16$ with a power level applied according to $E\left[\left|S_{k, l}^{p, p i l o t}\right|^{2}\right] / E\left[\left|S_{k, l}^{p}\right|^{2}\right]=10 \cdot \log _{10}\left(N_{p, \max }\right)$ (the fraction of power spent on pilots was always the same). Curves representing the false DER (false detection of users) and missed DER (users not detected) versus $E_{b} / N_{0}\left(E_{b}\right.$ is the average bit energy per packet and $N_{0}$ the one-sided power spectral density of channel noise) are shown in Fig. 2 for $N_{p, \max }=4$ and $P_{e}=0.5$. It is visible that for $E_{b} / N_{0}=2 \mathrm{~dB}$ the percentage of undetected users is already very low, around $0.2 \%$, and the percentage of false alarms is virtually inexistent.

To evaluate the proposed multipacket detection technique we used the analysis presented in [14] for a network-assisted diversity multiple access (NDMA) MAC protocol. It is assumed that the BS is responsible for running most of the calculations and to handle transmission collisions. When the BS detects collisions it requests $N_{p}-1$ retransmissions to all users using a broadcast control channel. We considered three scenarios: uncorrelated channel (UC), fixed channel (FC), which is a stationary scenario, and variable channel (VC) with mobile speeds of $30 \mathrm{~km} / \mathrm{h}$. An information block size of 3836 bits encoded with a rate-1/2 turbo code was employed resulting in a frame composed of 16 OFDM blocks. Fig. 3 , shows the impact of the symbol interleaving for different numbers of colliding packets in the block/packet error rate 


$$
p_{2}(y)=\frac{\left(\frac{y}{\mu_{2}}\right)^{N_{\text {pilots }}-1} \exp \left(-\frac{y}{(1-\sqrt{\rho}) \mu_{2}}\right)_{1} F_{1}\left(1, N_{\text {pilots }} ; \frac{\sqrt{\rho} N_{\text {pilots } y}}{(1-\sqrt{\rho})\left(1-\sqrt{\rho}+\sqrt{\rho} N_{\text {pilots }}\right) \mu_{2}}\right)}{\left(N_{\text {pilots }}-1\right) !(1-\sqrt{\rho})^{N_{\text {pilots }}-1}\left(1-\sqrt{\rho}+\sqrt{\rho} N_{\text {pilots }}\right) \mu_{2}} u(y)
$$

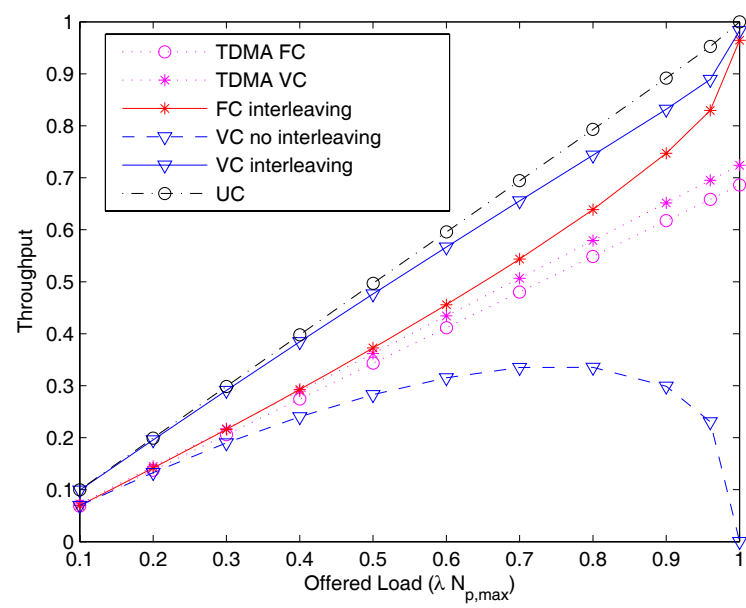

Fig. 4. Throughput when $E_{b} / N_{0}=4 \mathrm{~dB}$.

(BLER). Comparing the two VC scenarios we observe that the channel time correlation can preclude an efficient packet separation unless we employ different symbol interleavers for different retransmissions. The impact of the use of different symbol interleavers in the retransmissions is also clear in the FC scenario where we can still achieve reliable detection. In addition we can observe performance improvement as we increase $N_{p}$, which is a consequence of having adopted the $E_{b}$ for each packet (the total energy used to transmit a packet is $N_{p} E_{b}$ ). Due to size constraints we do not show but we verified that when comparing the results in Fig. 3, which employs the channel estimation approach described previously, with those obtained assuming perfect channel estimation there is only a minor performance loss. Fig. 4 shows the throughput versus offered load $\left(\lambda N_{p, \max }\right)$ for $E_{b} / N_{0}=4 \mathrm{~dB}$, which varies from very light load $(10 \%)$ until the saturation value $(100 \%)$ where all bandwidth is required. According to the results NDMA clearly outperforms TDMA for the conditions tested, especially for loads above $60 \%$, as long as symbol interleavers are used. Higher differences could be observed for lower $E_{b} / N_{0}$ values. The reason for this behavior is that our receiver can take full advantage of the overall energy spent to transmit the packet (i.e., the energy for all retransmission attempts). We would like to point out that although the throughput for high system load can be close to $100 \%$, the corresponding packet delay grows fast for large system loads, since the number of retransmission increases with the number of collisions, and the number of collisions is higher for higher loads. See (5) on top of the next page.

\section{CONCLUSIONS}

In this paper we considered a multipacket detection technique capable of dealing with MAC collisions in OFDM-based systems and achieve high throughputs. We showed that using different interleavers for different retransmissions our packet separation technique attains good performances even in slowvarying or constant channels. We also included a method to estimate the number of users involved in a collision.

\section{ACKNOWLEDGMENT}

The authors would like to acknowledge the anonymous reviewers for their helpful suggestions, which improved the quality of the paper. This work was partially supported by the FCT - Fundação para a Ciência e Tecnologia (pluriannual funding and U-BOAT project PTDC/EEA-TEL/67066/2006).

\section{REFERENCES}

[1] Y. Yu and G. Giannakis, "SICTA: a 0.693 contention tree algorithm using successive interference cancellation," in Proc. IEEE INFOCOM, Mar. 2005.

[2] X. Wang, Y. Yu, and G. Giannakis, "A roboust high-throughput three algorithm using successive interference cancellation," in Proc. IEEE GLOBECOM, Dec. 2005.

[3] M. Tsatsanis, R. Zhang, and S. Banerjee, "Network assisted diversity for random access wireless systems," IEEE Trans. Signal Process., vol. 48, pp. 702-711, Mar. 2000.

[4] R. Zhang and M. Tsatsanis, "Network-assisted diversity multiple access in time-dispersive channels," IEEE Trans. Commun., vol. 50, no. 4, pp. 623-632, Apr. 2002.

[5] G. Dimic, N. D. Sidiropoulos, and R. Zhang, "Signal processing and queueing tools for MAC-PHYS cross-layer design," IEEE Signal Process. Mag., special issue on signal processing and queueing aspects of network protocols, pp. 40-50, Sep. 2004.

[6] R. Dinis, P. Carvalho, L. Bernardo, R. Oliveira, M. Serrazina, and P.Pinto, "Frequency-domain multipacket detection: a high throughput technique for SC-FDE systems,"in Proc. IEEE GLOBECOM, Nov. 2007.

[7] L. Cimini, "Analysis and simulation of a digital mobile channel using orthogonal frequency division multiplexing," IEEE Trans. Commun., vol. 33, no. 7, July 1985.

[8] N. Souto, A. Correia, R. Dinis, J. C. Silva, and L. Abreu, "Multiresolution MBMS transmissions for MIMO UTRA LTE systems," in Proc. IEEE Int. Symp. Broadband Multimedia Syst. Broadcast., Mar. 2008.

[9] V. A. Aalo, "Performance of maximal-ratio diversity systems in a correlated Nakagami-fading environment," IEEE Trans. Commun., vol. 43, pp. 2360-2369, Aug. 1995.

[10] M.-S.Alouini, A. Abdi, and M. Kaveh, "Sum of gamma variates and performance of wireless communication systems over Nakagami-fading channels," IEEE Trans. Veh. Technol., vol. 50, no. 6, pp.1471-1480, Nov. 2001.

[11] M. Abramowitz and I. A. Stegun, Handbook of Mathematical Functions with Formulas, Graphs, and Mathematical Tables. Dover Publications, 1970.

[12] D. C. Montgomery and G. C. Runger, Applied Statistics and Probability for Engineers. John Wiley \& Sons, 1999.

[13] "Selection procedures for the choice of radio transmission technologies of UMTS," ETSI, TR 101112 v3.2.0, Sophia Antipolis, France, 1998

[14] N. Souto, R. Dinis, J. C. Silva, and P. C. Carvalho, "A high throughput technique for OFDM systems," in Proc. IEEE Wireless Commun. Networking Conf. (WCNC), Mar. 2008. 